\title{
Medicine at the 2000 Sydney Olympic Games: the New Zealand health team
}

A medical team of 14 accompanied the New Zealand team to the 2000 Olympics in Sydney. The team comprised four doctors, seven physiotherapists, a sports psychologist, a chiropractor, and a massage therapist. In addition, two teams (cycling and women's hockey) had dedicated physiotherapists, and sailing brought their own massage therapist.

The month long stay at the Games could be roughly divided into four one week periods. The first week involved the arrival of the medical team over the first weekend that the Games village was open. The sailing team arrived at a similar time and a few other athletes arrived during the week. This week was largely taken up with setting up the medical room in a bare basement area, reconnaissance of the village and Olympic site, attendance at training as required, and providing a service to those already in the village.

The second week, which was the week leading up to the opening ceremony, was very busy with the arrival of the remaining athletes including men's and women's basketball and women's hockey at the beginning of the week. Attendance at training and warm up games, continuing a day to day service to those in the village, and finishing off any last minute medical assessments and medicine notifications with respect to notifiable drugs. Each member of the New Zealand Olympic team was required to pass a medical before the beginning of the Games. Most of these were carried out in New Zealand in the month before departure for the Games. However, some of those who were based overseas presented for their medical on arrival in the village. The second week accounted for $42 \%$ of the total consultations (table 1).

The third week in the village was the first week proper of the Games, the clinic remained busy but the staff were rostered to attend events in support of athletes. Almost a third (31\%) of consultations were carried out in this week. Doctors were rostered to events according to a predetermined list of priorities-for example, dangerous sports, such as boxing, mountain biking, etc, and team sports. However, there was always one doctor available in the clinic. Physiotherapists also maintained a level of cover within the clinic as well as covering sports.

In the final week at the Games, the New Zealand team had fewer athletes competing as the team sports came to the end of their competitions, and subsequent presentations at the clinic were reduced. The final two days were set aside to arrange for medical and physiotherapy supplies to be left for the Paralympic team.

During the period of the Games, the medical team were able to make use of the superb imaging facilities provided by Dr Jock Anderson and his colleagues at the village polyclinic. Readers may be interested to know that three of our athletes with nerve root impingement had computed tomography guided nerve root sleeve injections. All three were able to resume full training within 72 hours. Of the 150 athletes in the New Zealand team, only one was unable to participate in his chosen event on account of injury.

The medical clinic was open 8 am to 10 pm each day with an on call doctor available overnight. Apart from the dedicated physiotherapists and massage therapists as outlined above, men's and women's basketball and women's softball had nominated physiotherapists from within the medical team who provided most of their care and game coverage. The other physiotherapists also looked after athletes with whom they had had previous contact. However, overall the doctors provided general team coverage and rotated game coverage at team sports. The feedback from the athletes was generally positive in that they felt comfortable with being able to approach and be attended by any of the four doctors.

In summary, a multidisciplinary medical team provided health care to 150 athletes and 70 officials during the 2000 Sydney Olympic games. The peak workload occurred in the week immediately preceding competition. Prioritising events ensured that we made the most appropriate use of limited resources. For a team the size of New Zealand's, appointing most health team members as headquarters staff allowed flexibility in assigning them to a variety of teams and events.

D Robinson

Sportsmed Canterbury, 156 Bealey Avenue, Christchurch, New Zealand

C Milne

87 Clarence Street, Hamilton, New Zealand

Correspondence to: Dr Robinson; info@sportsmed-nz.co.nz

Table 1 Consultations of the New Zealand team at the 2000 Sydney Olympics

\begin{tabular}{|c|c|c|c|}
\hline \multicolumn{4}{|c|}{ 1. Medical consultations } \\
\hline \multicolumn{2}{|c|}{ Athlete } & 493 & \\
\hline \multicolumn{2}{|c|}{ Official (manager, coach, headquarters, medical) } & 106 & \\
\hline \multicolumn{2}{|c|}{ Other (NZ house staff, sponsor) } & 7 & \\
\hline \multicolumn{2}{|c|}{ Total } & 606 & \\
\hline \multicolumn{4}{|l|}{ Presentations } \\
\hline \multicolumn{2}{|l|}{ Injury } & \multicolumn{2}{|l|}{ Medical } \\
\hline Head & 2 & Respiratory tract infection & 101 \\
\hline Neck & 18 & Respiratory other, eg asthma & 15 \\
\hline Shoulder & 28 & Gastrointestinal infection & 13 \\
\hline Elbow & 11 & Gastrointestinal other & 13 \\
\hline Forearm & 4 & Headache & 5 \\
\hline Wrist & 16 & Eye & 14 \\
\hline Hand & 18 & Ear/nose & 6 \\
\hline Thoracic spine & 4 & Mouth/dental & 14 \\
\hline Lumbar spine & 49 & Skin & 49 \\
\hline Chest wall & 7 & Cardiac & 1 \\
\hline Abdomen & 0 & Urinary tract infection & 2 \\
\hline Hip, groin & 28 & Gynaecological & 10 \\
\hline Thigh & 20 & Diet/weight control advice & 8 \\
\hline Knee & 57 & Other infection & 4 \\
\hline Lower leg & 9 & Allergy & 11 \\
\hline Ankle, achilles & 41 & Other, eg insomnia, fatigue & 20 \\
\hline Foot & 33 & Medicals, medication notification & 22 \\
\hline Total & 345 & Total & 308 \\
\hline \multicolumn{4}{|c|}{ NB: Some consultations involved more than one presenting problem } \\
\hline \multicolumn{2}{|c|}{ 2. Physiotherapy consultations } & Total & 1403 \\
\hline \multicolumn{2}{|c|}{ 3. Chiropractic consultations (includes acupuncture) } & Total & 286 \\
\hline
\end{tabular}

